\title{
Names of Bacteria Validly Published in 1980
}

The names in the following list were validly published in 1980, either by publication in an original paper in the IJSB or, if published elsewhere, by announcement in the IJSB. Each bacterial name is followed by the name(s) of the author(s) responsible for the name and then by the citation to the publication of the name in the IJSB. For each year, a list of names validly published in that year will be published in the January issue of the following year. Cumulative lists will be published every 5 years. Consequently, one will be able easily to discern those bacterial names which have nomenclatural standing at any given time: one need merely to consult the Approved Lists of Bacterial Names (Int. J. of Syst. Bacteriol. 30:225-420, 1980), the latest annual list, and the issues of the IJSB published in the same year as the latest annual list. That is to say, since the cumulative list for 1981 will not be published until January 1982, at any time in 1981, one would also have to search the 1981 issues of the IJSB which had been published up to the time of the search.

The Editor

Acetivibrio Patel et al. 1980 (30:179)

Acetivibrio cellulolyticus Patel et al. 1980 (30:179)

Acholeplasma morum Rose et al. 1980 (30:653)

Actinomadura macra Huang 1980 (30:565)

Bacteroides gingivalis Coykendall et al. 1980 (30:563)

Bacteroides macacae (Slots and Genco) Coykendall 1980 (30:563)

Bacteroides melaninogenicus subsp. macacae Slots and Genco 1980 (30:84)

Beneckea gazogenes Harwood et al. 1980 (30:655)

Beneckea nereida Harwood et al. 1980 (30:655)

Cellulomonas cartae Stackebrandt and Kandler 1980 (30:186)

Chromatium purpuratum Imhoff and Trüper 1980 (30:601)

Corynebacterium liquefaciens (Okabayashi and Masuo) Lanéelle et al. 1980 (30:544)

Corynebacterium vitarumen (Bechdel et al.) Lanéele et al. 1980 (30:542)

Cytophaga heparina (Payza and Korn) Christensen 1980 (30:475)

Enterobacter gergoviae Brenner et al. 1980 (30:1)

Enterobacter intermedium Izard et al. 1980 (30:601)

Enterobacter sakazakii Farmer et al. 1980 (30:569)

Eubacterium brachy Holdeman et al. 1980 (30:164)

Eubacterium nodatum Holdeman et al. 1980 (30:164)

Eubacterium timidum Hol̀deman et al. 1980 (30:164)

Flexibacter canadensis Christensen 1980 (30:431)

Fluoribacter Garrity et al. 1980 (30:612)

Fluoribacter bozemanae Garrity et al. 1980 (30:612)

Frateuria Swings et al. 1980 (30:555)

Frateuria aurantia Swings et al. 1980 (30:555)

Gardnerella Greenwood and Pickett 1980 (30:176)

Gardnerella vaginalis (Gardner and Dukes) Greenwood and Pickett 1980 (30:176)

Halomonas Vreeland et al. 1980 (30:494)

Halomonas elongata Vreeland et al. 1980 (30:495)

Lactobacillus bavaricus Stetter and Stetter 1980 (30: 601)

Lactobacillus gasseri Lauer and Kandler 1980 (30: 601)

Legionella bozemanii Brenner et al. 1980 (30:676)
Legionella dumoffii Brenner et al. 1980 (30:676)

Legionella gormanii Morris et al. 1980 (30:676)

Legionella micdadei Hebert et al. 1980 (30:676)

Legionella pittsburghensis Pasculle et al. 1980 (30: 676)

Methylococcus mobilis Hazeu et al. 1980 (30:676)

Mycobacterium sphagni Kazda 1980 (30:81)

Mycoplasma fastidiosum Lemcke and Poland 1980 (30:161)

Photobacterium logei Harwood et al. 1980 (30:655)

Planctomyces maris Bauld and Staley 1980 (30:595)

Prosthecobacter Staley et al. 1980 (30:595)

Prosthecobacter fusiformis Staley et al.1980 (30:595)

Pseudomonas carboxydohydrogena Meyer et al. 1980 (30:194)

Renibacterium Sanders and Fryer 1980 (30:501)

Renibacterim salmoninarum Sanders and Fryer 1980 (30:501)

Spirochaeta aurantia Canale-Parola 1980 (30:594)

Spirochaeta litoralis Canale-Parola 1980 (30:594)

Spirochaeta zuelzerae Canale-Parola 1980 (30:595)

Sulfolobus brierleyi Zillig et al. 1980 (30:676)

Sulfolobus solfataricus Zillig et al. 1980 (30:676)

Tatlockia Garrity et al. 1980 (30:612)

Tatlockia micdadei (Hebert et al.) Garrity et al. 1980 (30:612)

Treponema bryantii Stanton and Canale-Parola 1980 (30:676)

Vampirovibrio Gromov and Mamkayeva 1980 (30: 676)

Vampirovibrio chlorellavorus Gromov and Mamkeyeva $1980(30: 676)$

Vibrio vulnificus Farmer 1980 (30:656)

\section{List of Specific and Subspecific Epithets}

aurantia (Frateuria, Spirochaeta)

bavaricus (Lactobacillus)

bozemanae (Fluoribacter)

bozemanii (Legionella)

brachy (Eubacterium)

brierleyi (Sulfolobus) 
bryantii (Treponema)

canadensis (Flexibacter)

carboxydohydrogena (Pseudomonas)

cartae (Cellulomonas)

cellulolyticus (Acetivibrio)

chlorellavorus (Vampirivibrio)

dumoffii (Legionella)

elongata (Halomonas)

fastidiosum (Mycoplasma)

fusiformis (Prosthecobacter)

gasseri (Lactobacillus)

gazogenes (Beneckea)

gergoviae (Enterobacter)

gingivalis (Bacteroides)

gormanii (Legionella)

heparina (Cytophaga)

intermedium (Enterobacter)

liquefaciens (Corynebacterium)

litoralis (Spirochaeta)

logei (Photobacterium) macacae (Bacteroides, Bacteroides melaninogenicus subsp.)

macra (Actinomadura)

maris (Planctomyces)

micdadei (Legionella, Tatlockia)

mobilis (Methylococcus)

morum (Acholeplasma)

nereida (Beneckea)

nodatum (Eubacterium)

pittsburghensis (Legionella)

purpuratum (Chromatium)

sakazakii (Enterobacter)

salmoninarum (Renibacterium)

sphagni (Mycobacterium)

solfataricus (Sulfolobus)

timidum (Eubacterium)

vaginalis (Gardnerella)

vitarumen (Corynebacterium)

vulnificus (Vibrio)

zuelzerae (Spirochaeta) 